\title{
Analysis of the dynamics of limb transcriptomes during mouse development
}

\author{
Istvan Gyurján ${ }^{1}$, Bernhard Sonderegger ${ }^{1}$, Felix Naef ${ }^{1,2}$ and Denis Duboule ${ }^{1,3^{*}}$
}

\begin{abstract}
Background: The development of vertebrate limbs has been a traditional system to study fundamental processes at work during ontogenesis, such as the establishment of spatial cellular coordinates, the effect of diffusible morphogenetic molecules or the translation between gene activity and morphogenesis. In addition, limbs are amongst the first targets of malformations in human and they display a huge realm of evolutionary variations within tetrapods, which make them a paradigm to study the regulatory genome.

Results: As a reference resource for future biochemical and genetic analyses, we used genome-wide tiling arrays to establish the transcriptomes of mouse limb buds at three different stages, during which major developmental events take place. We compare the three time-points and discuss some aspects of these datasets, for instance related to transcriptome dynamics or to the potential association between active genes and the distribution of intergenic transcriptional activity.

Conclusions: These datasets provide a valuable resource, either for research projects involving gene expression and regulation in developing mouse limbs, or as examples of tissue-specific, genome-wide transcriptional activities.
\end{abstract}

\section{Background}

Limb development has fascinated biologists for a century, mostly because of the importance of these structures in the evolution of land vertebrates and due to their spectacular morphological diversity. From an experimental viewpoint, limbs are quite easily accessible at various stages of their ontogenesis and can thus be manipulated, to some extent. Genetically speaking, limb phenotypes can be easily detected and usually do not impair survival too strongly. For all these reasons, limbs have been excellent model structures to study vertebrate patterning and morphogenesis.

Tetrapods limbs bud out from the lateral plate mesoderm and establish early on the bases of a three-dimensional pattern. The growth along the proximo-distal axis largely depends on FGFs signaling emanating from the apical ectodermal ridge (AER) and acting over the mesenchyme. On the other hand, the anterior to posterior (AP) axis is specified essentially by Hand2, the Shh pathway and posterior Hoxd genes. Amongst the known

\footnotetext{
* Correspondence: denis.duboule@unige.ch

'School of Life Sciences, Ecole Polytechnique Fédérale, Station 19, Lausanne, 1215 Switzerland

Full list of author information is available at the end of the article
}

regulators of the dorso-ventral (DV) patterning are Wnt7a, expressed in dorsal ectoderm, and Engrailed, in ventral ectoderm, as well as $L m x b 1$, a gene transcribed in dorsal mesenchyme. These signaling cascades act together in a highly coordinated manner [1-3].

At later stages of development, e.g. starting from E11.5 onwards in mice, mesenchymal condensations form and establish the future skeletal pattern, following a tightly regulated process. The first elements to appear are those of the future stylopod (humerus, femur), then of the zeugopod (radius, ulna, tibia, fibula) and, finally, the autopod (hands and feet). This period of limb development, referred to as 'proliferative expansion, determination and differentiation' [2], is a very dynamic phase, where differences amongst tetrapods start to be translated from the genetics (the transcription, maintenance and silencing of regulatory genes and their readouts) to species-specific morphologies. Such differences in the shapes of tetrapod limbs, may rely upon slight variations in these 'genetic' parameters, such as transcriptional heterochronies or quantitative effects of key developmental molecules [4-6].

While many of those genes critical for limb development were described over the past 15 years, additional
C Biomed Central

C 2011 Gyurján et al; licensee BioMed Central Ltd. This is an Open Access article distributed under the terms of the Creative Commons Attribution License (http://creativecommons.org/licenses/by/2.0), which permits unrestricted use, distribution, and reproduction in any medium, provided the original work is properly cited. 
players, such as regulatory RNAs, have come into play more recently. There is indeed increasing evidence that long non-coding RNAs (ncRNAs) may be crucial for some developmental processes [7] due to their involvement in various functions including gene regulation, both at the transcriptional and post-transcriptional levels, in the organization of epigenetic modifications [8] as well as during gene activation and silencing [9]. Surprisingly, inter-species sequence conservation does not seem to be essential [10], even in the cases of the Xist, Air or $E v f-2$ RNAs where important functions were demonstrated [11-13]. The best-studied long ncRNAs are associated with imprinted gene clusters, where many of them act by repressing neighboring genes via a ciseffect $[13,14]$.

As a resource for research projects involving gene expression and regulation in developing mouse limbs, we set up to produce and analyze whole genome expression data for E11.5, E13.5 and E15.5 forelimbs. We selected these three stages of embryonic limb development not only because these days are very dynamic, in terms of growth and organogenesis, but also because the transcriptional activation of several known genes occurs during this time-period, giving us both an idea of the relevance of the datasets, and the possibility to look at the vicinities of these loci to evaluate a potential clustering of transcriptional activities. Within this time-window indeed, the muscular and skeletal systems develop in parallel with massive vascularization, innervations and skin formation, implying large amounts of new cell types and tissue interactions. We selected three different ontogenetic stages to see how global transcriptional activity evolves along with development of a complex structure, not only by considering protein coding genes, but also intergenic transcription and non-coding RNA expression.

\section{Results and discussion}

We sampled mouse embryonic forelimbs at days 11.5, 13.5 and 15.5 postcoïtum, by cutting off the entire forelimb buds from the body wall (Figure 1A). The particular morphogenetic events occurring at these stages have been largely described earlier (e.g. [15]). Briefly, E11.5 limb buds consist of a rather poorly differentiated inner mesenchyme, located within an epidermal envelope. Despite this apparent uniformity, mesenchymal cells at this stage can be already differentiated as belonging to denser, centrally located regions where condensation of the future cartilage rods starts to occur [2]. These condensations appear with a time sequence that follows a proximal to distal progression. At the same stage, the first nerve fascicles become apparent, originating from the brachial plexus.

By day 13.5, the cartilage primordia of the future limb skeleton are already well defined, the digits start to

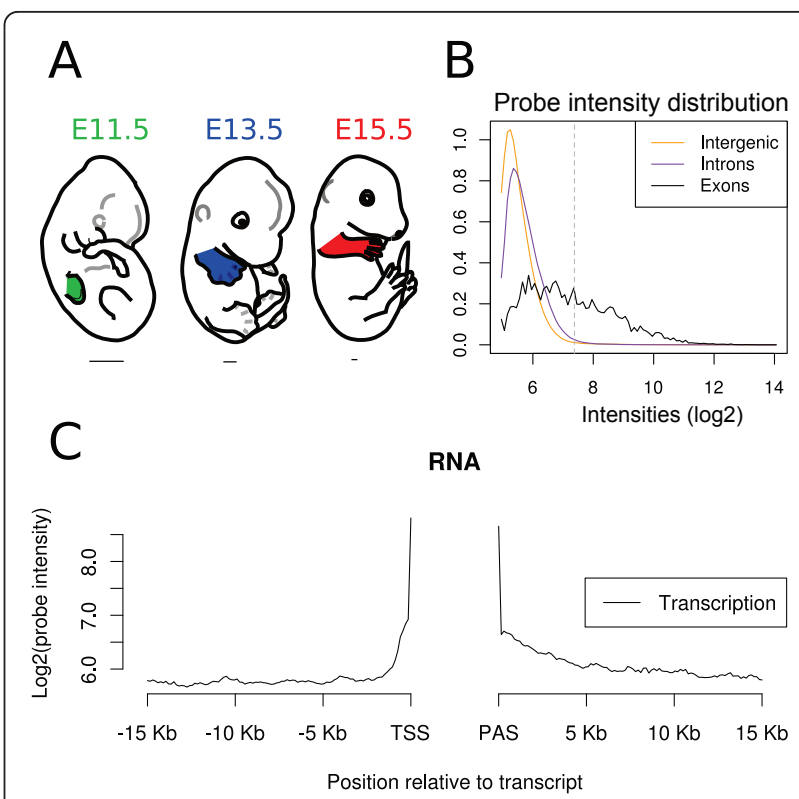

Figure 1 Experimental setup and tiling array intensity distributions. A: Schematics of embryos at the three stages of limb development (E11.5, E13.5 and E15.5) considered in this study. Forelimbs are indicated with a color code that is used in subsequent figures. The bars below the embryos indicate relative size differences. B: Histogram showing the intensity distributions along chromosome 2 for intergenic, intronic and exonic transcription. Exons considered are as defined in UCSC Known Genes annotation. C: Distribution of probe intensity around expressed genes on chromosome 2. TSS: transcription start site; PAS: polyadenylation site. Vertical gray dotted line: determined threshold level (in this example $\log 2=7.554$ ).

separate from one another as a result of inter-digital cell death. There is massive deposition of muscle elements (already initiating in late E11.5 buds), nerves extend into the hand plates and few hair buds are already apparent. The limbs are fully vascularised and the skin starts to stratify. Embryonic forelimbs at day 15.5 have completely separated digits. The ossification process, which starts around E14.0, is in progress even in the most distal phalanges. Most of the tendons are now well defined and there is strong progression of skin stratification.

\section{Expression signals are frequently confined to known transcript boundaries but tend to extend past the 3 'ends of genes}

Following RNA extraction and processing (see materials and methods) labelled cDNAs were hybridized onto whole genome tiling arrays (Affymetrix, Genechip Mouse Tiling 1.1R). After data normalization (see materials and methods) we used the UCSC Known Gene annotation system $(\mathrm{mm} 9,2007)$ to quantify expression levels in exons, introns and intergenic regions. Intensity 
thresholds to determine expressed transcripts and genes were calculated for each array, as exemplified by using chromosome 2 (Figure 1B). Distributions showed low intensities both in introns and intergenic regions, with introns showing slightly higher signals possibly reflecting precursor mRNAs or splice forms not covered by our annotation. In contrast the distributions for exons were clearly shifted towards higher expression. We also observed a bias in intensity around annotated transcripts since the signals in regions downstream of the annotated transcript ends were usually more pronounced and decayed more slowly than in regions upstream of the transcription start sites (TSSs). Also, in several cases, the 3' untranslated regions (UTR) were much longer than anticipated from the UCSC Known Gene annotation (Figure 1C). Overall, we observed that transcription was mostly confined to the vicinity of annotated genes, a finding that is consistent with RNA-seq studies in mouse and human [16], though some novel transcription sites were found (see below).

\section{About $40 \%$ of known genes are expressed at E11.5, E13.5 and E15.5}

The global expression data for individualized exons, transcripts and genes at the three developmental stages are shown in Table 1 (panel A). 41.17\%, 42.69 and $44.25 \%$ of known genes were expressed in forelimbs at E11.5, E13.5 and E15.5, respectively. Based on UCSC gene models, about $90 \%$ of these were annotated as coding genes (we incorporated 'near-coding genes' into this category). Interestingly about $95 \%$ of all expressed genes were common to the three developmental stages, with an average increase of $4 \%$ between E11.5 towards E15.5 limbs (Figure 2).

\section{Differential gene expression}

We compared the expressed genes between the three developmental stages to evaluate which percentage was significantly changed along with limb development (see Methods). Between E11.5 and E13.5, we identified 601 upregulated genes and 388 down-regulated genes (Figure 2, Additional file 1). Between E13.5 and E15.5 forelimbs, 924 genes were found up-regulated and 355 down-regulated. While these figures indicate that the general tendency goes towards an increase in the number of genes expressed, along with developmental time, they should be considered carefully since they derive from comparisons between steady-state levels of RNAs. Consequently, they do not necessarily reflect transcriptional switches and may point to RNAs whose transcription has increased, in the case of a gene considered as 'newly expressed'. Likewise, RNA stability may prevent, in some cases, the timely detection of a particular transcriptional switch-off. In addition, the cellular topographies of gene expression patterns are usually not homogenous and hence variations in relative transcript abundance may also indicate variations in space rather than in time (see below).

We looked into these parameters by quantitative PCRs on some of the genes, for which changes in expression patterns had been previously reported (Additional file 2). In addition, six genes with either known or unknown expression profiles, were validated by in situ hybridization (Additional file 2). As examples, the $C b \ln 1$ mRNA, essential during synapse formation [17] yet with previously unknown profile, was weakly expressed at E11.5 only, whereas the Tmem $8 c$ gene was specifically expressed in early muscle elements along both the trunk and the limb and progressively increased its expression throughout development.

Table 1 Summary of gene expressed during the development of mouse embryonic limb at the three developmental stages studied

\begin{tabular}{|c|c|c|c|c|c|}
\hline \multicolumn{3}{|c|}{ Developmental stage } & \multirow{2}{*}{$\begin{array}{c}\text { E.11.5 } \\
45.45 \%(102020 / 224464)\end{array}$} & \multirow{2}{*}{$\begin{array}{c}\text { E13.5 } \\
47.01 \%(105530 / 224464)\end{array}$} & \multirow{2}{*}{$\begin{array}{c}\text { E15.5 } \\
48.65 \%(109203 / 224464\end{array}$} \\
\hline A & $\begin{array}{c}\text { UCSC } \\
\text { known gene }\end{array}$ & Exons & & & \\
\hline & & Transcripts & $24.91 \%(11500 / 46163)$ & $25.99 \%(12000 / 46163)$ & $26.23 \%(12113 / 46163)$ \\
\hline & & Genes & $41.17 \%(10074 / 24469)$ & $42.69 \%(10446 / 24469)$ & 44.25\% (10828/24469) \\
\hline & Gene models & Coding & $90.71 \%(9139 / 10074)$ & $90.5 \%(9454 / 10446)$ & $91.35 \%(9892 / 10828)$ \\
\hline & & Antisense & $2.29 \%(231 / 10074)$ & $2.42 \%(253 / 10446)$ & $2.25 \%(244 / 10828)$ \\
\hline & & Non-coding & $6.98 \%(704 / 10074)$ & $7.07 \%(739 / 10446)$ & 6.39\% (692/10828) \\
\hline B & Genes with intronic & gene is expressed) & $4841(3296)$ & $5155(3662)$ & 4660 (3259) \\
\hline \multirow[t]{5}{*}{$\mathrm{C}$} & \multicolumn{2}{|c|}{ Intergenic regions } & 7408 & 7471 & 7477 \\
\hline & \multicolumn{2}{|c|}{ Directly flanking UCSC genes } & $528(7.13 \%)$ & $526(7.04 \%)$ & $537(7.18 \%)$ \\
\hline & \multicolumn{2}{|c|}{ Identifiable via ENSEMBL } & $1981(26.74 \%)$ & $1970(26.37 \%)$ & 1994 (26.67\%) \\
\hline & \multicolumn{2}{|c|}{ Directly flanking ENSEMBL genes } & $587(7.92 \%)$ & $576(7.71 \%)$ & $592(7.92 \%)$ \\
\hline & \multicolumn{2}{|c|}{ Unexplained } & $4312(58.21 \%)$ & 4399 (58.88\%) & $4354(58.23 \%)$ \\
\hline
\end{tabular}

A: UCSC Known Gene expression and gene model categories. B: Intronic transcription. Numbers in parentheses shows those intronic transcripts for which the genes containing them were also expressed. C: Transcription of intergenic regions and sub-categories (see Materials and Methods). The 'unexplained' category represents transcribed intergenic regions remaining after filtering (cf. Methods). 


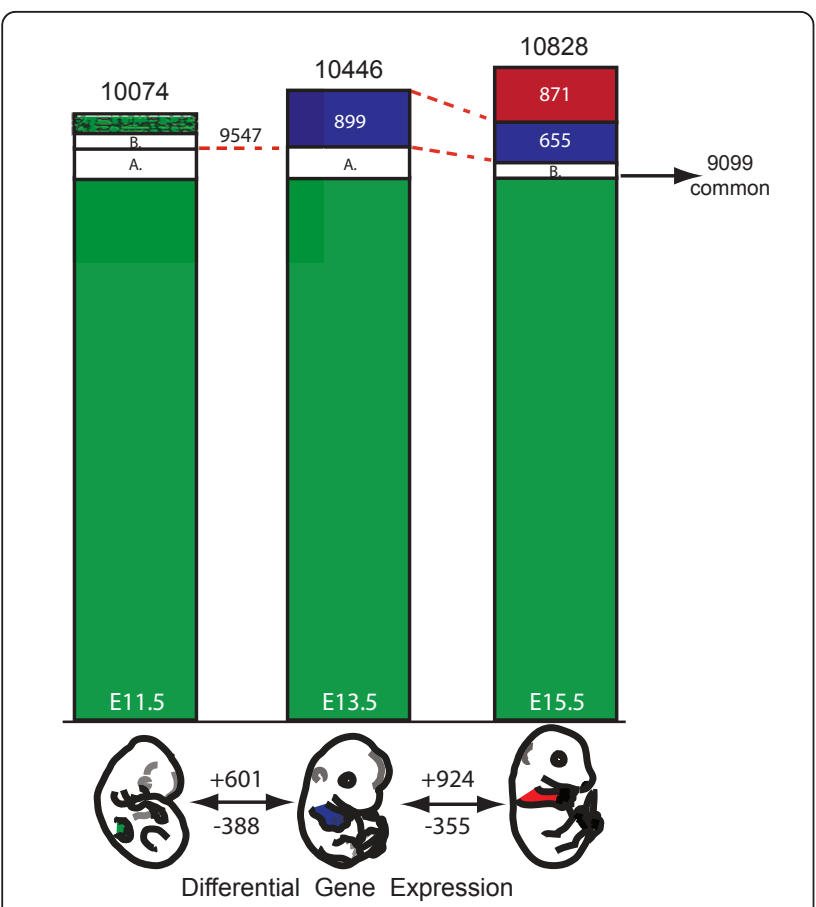

Figure 2 Gene expression dynamics during three stages of limb development. Top: The expressed UCSC Known Genes at the three limb developmental stages. Smooth green areas represent genes expressed at all three stages $(n=9099)$. Blue areas show genes whose transcription is gained at E13.5 $(n=899)$ and still found expressed in embryos at E15.5 $(n=655)$. The red area represents genes that are significantly expressed only at E15.5 ( $n=$ 871). The patterned green area in E11.5 highlights those genes that are expressed only at E11.5 $(n=297)$. The white areas indicate genes that are expressed in both E11.5 and E13.5 limbs, but not at E15.5 $(\mathbf{A} ; n=475)$, or with genes expressed in both E11.5 and E15.5 (B; $n=203$ ), yet not in E13.5 limbs. Bottom: Differential gene expression in forelimbs between the developmental stages. The numbers of up- and down-regulated genes are indicated above and below the arrows, respectively.

To evaluate whether differentially expressed genes could be grouped into particular functional categories, we used the Ingenuity Knowledge Base http://www.ingenuity.com. This analysis indicated that functional categories such as muscle and skeletal system development, or skin development were enriched, as expected from the developmental stages under scrutiny (Table 2). We also performed a more detailed gene ontology analyses using GOMiner [18], to see if development related terms were enriched in our sets of differentially expressed genes. Functions were clustered into hierarchical trees (see Additional files 3 and 4) and the results showed that terms related to muscle development again dominated, both when E11.5 limbs were compared with E13.5, and when E13.5 limbs were compared with E15.5. This is most likely due to high number of genes encoding muscle structural proteins. Cartilage, bone, and cell- adhesion related term enrichments were also scored. Noteworthy, the number of functional clusters was higher between E13.5 and E15.5 than between the two younger stages, which reflects an increased tissue diversification along with the processing of limb development. We matched our sets of differentially expressed genes with the mammalian phenotype database http:// www.informatics.jax.org and scored 69 genes that had been previously associated with abnormal or short limb morphology (Additional file 5).

\section{Intronic and intergenic transcription}

Although we applied a rather stringent threshold, we observed extensive intronic transcription, since about 50 percent of expressed genes also contained signals $(\geq 300$ bp) covering intronic sequences (Table 1B). While, in some instances, this can be explained by introns retained after splicing, the majority of this intronic activity seems to derive either from alternative start sites, as suggested by looking at some profiles of histone posttranslational modification tracks from various cell types in the UCSC Genome Browser [19,20], or from alternative transcription termination, which seems to occur frequently in human or mouse genes and can thus lead to intronic transcription [21]. In addition, about 30 percent of these intronic signals were detected within genes that are not expressed during limb development, indicating the presence of independent transcription units overlapping with known genes.

We also scored 7408,7471 and 7477 transcribed regions above threshold ( $\geq 300 \mathrm{bp}$ ), at E11.5, E13.5 and E15.5, respectively, within intergenic regions (Table 1C). We further filtered this raw data using the Ensembl database for possible overlaps and approximately 25 percent of these sequences were thus identified. Half of these matched protein coding genes, whereas the other half was composed of pseudogenes and retrotransposed elements (Additional file 6). Protein coding genes showed 48 percent parity with UCSC coding genes which, due to different annotation of 3'UTR sequences, and hence because of their different lengths, had not been identified earlier. After this filtering, 4312, 4399 and 4354 intergenic regions remained clearly transcribed during the three developmental stages, respectively (hereafter termed the 'unexplained' category). Some of these regions may reflect alternative start sites, extended 3'UTRs, retrotransposed or pseudogene elements, as well as non-coding RNAs.

We next asked whether these transcribed intergenic regions were distributed randomly throughout the genome or, alternatively, whether they would tend to be associated with (or be located at the vicinity of-) active (or silent) gene loci. We thus analyzed the transcriptional status of those genes located around each 
Table 2 Ingenuity top five categories of differentially expressed genes concerning the development of physiological system

\begin{tabular}{lcc}
\hline $\begin{array}{l}\text { Differential Gene Expression: E13,5 vs. E11,5 } \\
\text { Name }\end{array}$ & p-value* & Number of Molecules \\
\hline Tissue Development & $6,56 E-14-4,42 \mathrm{E}-03$ & 148 \\
Skeletal and Muscular System Development and Function & $4,51 \mathrm{E}-11-4,42 \mathrm{E}-03$ & 160 \\
Organ Development & $4,87 \mathrm{E}-11-4,03 \mathrm{E}-03$ & 137 \\
Cardiovascular System Development and Function & $1,35 \mathrm{E}-08-4,42 \mathrm{E}-03$ & 92 \\
Tissue Morphology & $9,38 \mathrm{E}-08-4,42 \mathrm{E}-03$ & 138 \\
\hline Differential Gene Expression: E15,5 vs. E13,5 & $\mathbf{p}-$-value* & Number of Molecules \\
Name & $2,06 \mathrm{E}-19-1,45 \mathrm{E}-03$ & 239 \\
\hline Skeletal and Muscular System Development and Function & $2,06 \mathrm{E}-19-1,13 \mathrm{E}-03$ & 217 \\
Tissue Morphology & $6,00 \mathrm{E}-16-1,42 \mathrm{E}-03$ & 283 \\
Tissue Development & $2,63 \mathrm{E}-11-1,35 \mathrm{E}-03$ & 205 \\
Hematological System Development and Function & $3,28 \mathrm{E}-11-7,80 \mathrm{E}-03$ & 74 \\
Hair and Skin Development and Function & & \\
\hline
\end{tabular}

*Fisher's exact tests were used to calculate p-values.

transcribed intergenic region, to assess whether they were themselves expressed or not (Figure 3). Our genome-wide survey indicates that transcribed intergenic regions' are indeed found more frequently at the vicinity of transcribed genes, an observation valid as long as a distance of less than about $50 \mathrm{~kb}$ is considered. If a larger distance is allowed, this association becomes less significant (Figure 3A). The same tendency was observed when we estimated the fraction of expressed, or nonexpressed genes, with flanking transcribed intergenic regions (Figure 3B-D). There again, a higher fraction of expressed genes, as compared to non-expressed, showed flanking intergenic transcription. In this case, interestingly, this correlation was increased when considering the sub-group of genes coding for transcription factors (Figure 3B,C) and even non-expressed transcription factor-coding genes appeared to be more frequently surrounded by transcribed intergenic regions.

These results may reflect the presence, in the vicinity of annotated genes, of alternatively spliced regions, or alternate start sites, located at a distance, which had not been previously identified. It may also indicate that intergenic transcription may result from the nearby transcription of a 'standard' transcription unit, via a generic by-stander effect. For example, modifications of the epigenetic landscape associated with gene transcription may generate ectopic transcripts in the neighbourhood. In support of this, the fact that this observation is accentuated around transcription factors, i.e. nearby genes that, at least during development, are often controlled by remote regulations, over large distances [22]. We also measured the frequency of intergenic signals around those genes up-regulated between E11.5 and E15.5. However, the number of such genes with associated intergenic signals within a given distance was too low to be fully significant. The fact that transcription factor genes scored as 'not-expressed' showed an aboveaverage occurrence of intergenic signal around them raises the possibility that these loci may maintain 'open' local chromosomal domains for subsequent activation, as proposed in the case of embryonic stem (ES) cells based on the existence of a bivalent state in chromatin modifications [23].

\section{Differentially expressed ncRNAs}

We searched for a differential expression of non-coding RNAs (ncRNAs) annotated in UCSC, between the three developmental stages. After careful (manual) curation, which removed all potential 3'UTRs, pseudogenes and non-coding isoforms of coding genes, we identified 49 long ncRNAs that were differentially expressed in our samples (Table 3). Amongst them, ten were typical bidirectional transcripts, one was a cis-antisense transcript, two were antisense in the 3' end of genes (tail-totail), whereas the remaining ncRNAs were not closely associated with any gene. Twenty-two ncRNAs increased their relative amounts at steady state levels, between E11.5 and E13.5, whereas eleven of them decreased in amount between E11.5 and E13.5. Between E13.5 and E15.5, eleven ncRNAs were up-regulated and 25 down-regulated. As an example, the ncRNA Neat1 appeared as progressively up-regulated, consistent with the earlier observation that Neat 1 expression was reenforced during muscle cell differentiation [24]. We also assessed the level of conservation of these transcripts (using PhasCons scores) and found that they were globally poorly conserved in sequences amongst vertebrates, in agreement with the idea that the transcription itself of these RNAs or their structure, rather than sequence conservation, may be important $[25,10]$. 


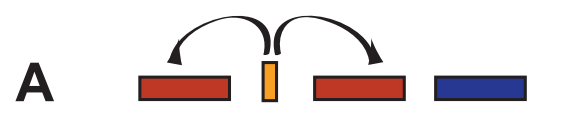

Distribution of intergenic signal

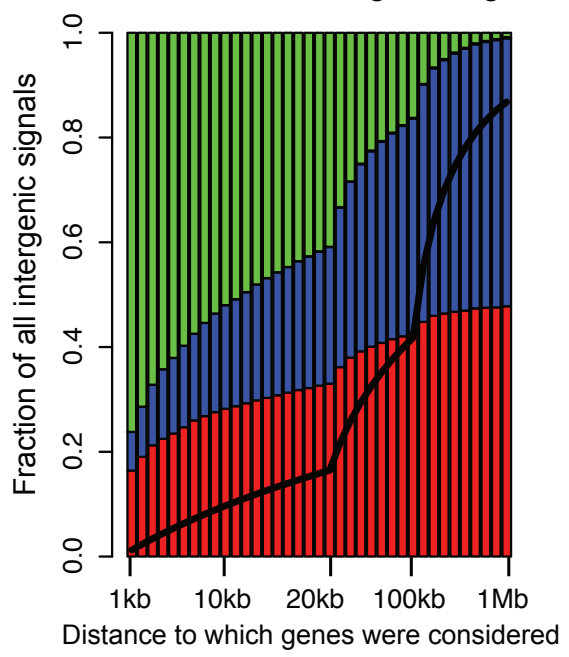

E11.5
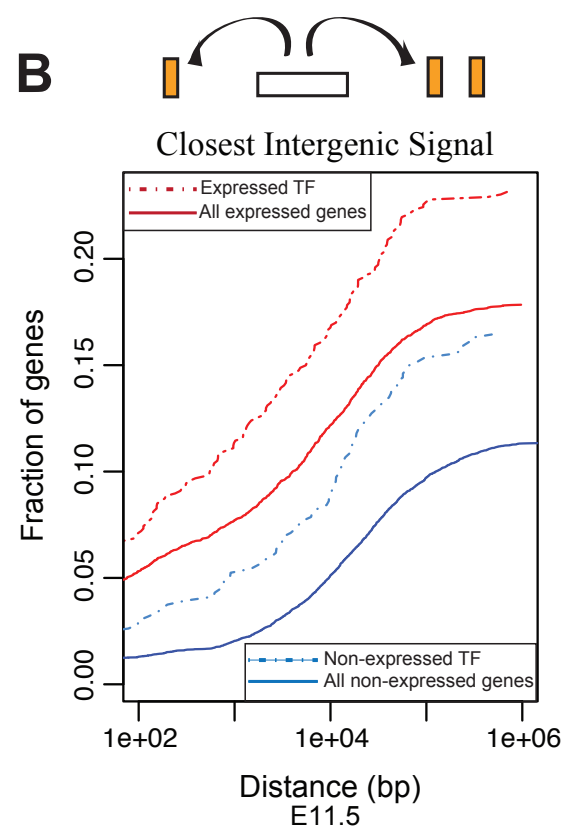

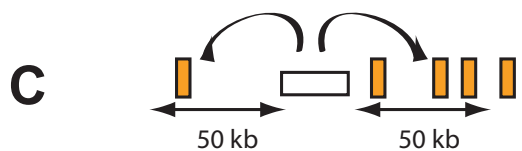

Density of intergenic signals

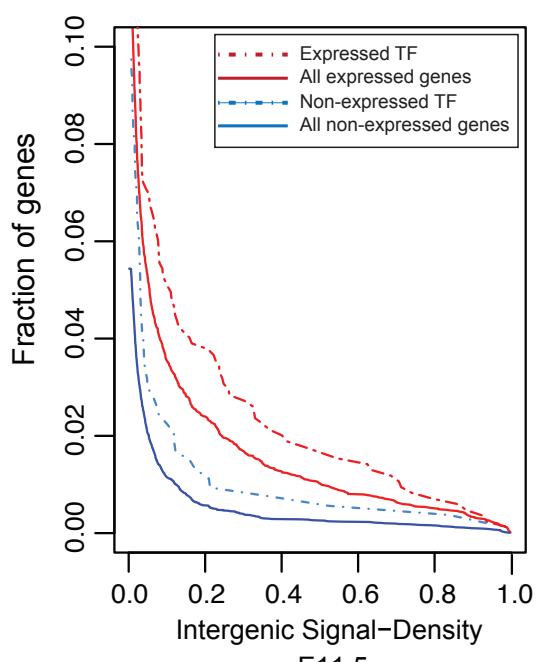

E11.5
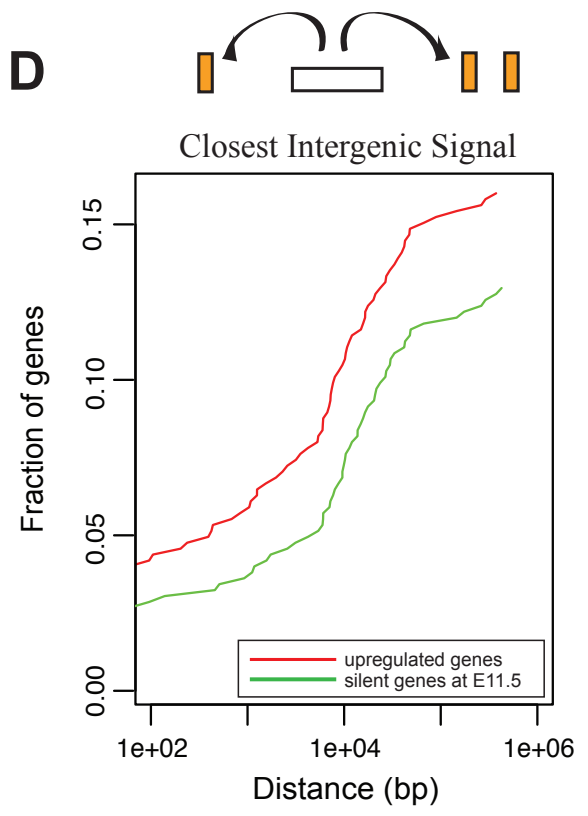

Figure 3 Intergenic transcription and proximity to expressed genes. A: All 4312 expressed intergenic regions (orange) at E11.5 were annotated as 'close to' an expressed gene (red), 'close to' a non-expressed (blue), 'not close to' a gene (green). Black lines show the expected borders between blue and green areas if genes were distributed randomly relative to expressed intergenic regions. B: Expressed genes are enriched with nearby intergenic transcription. Fraction of genes (expressed, solid red line $N=8582$; or not-expressed, solid blue line $N=14848$ ) with an expressed intergenic region within $\mathrm{x}$ bp is plotted over genomic distance ( $\mathrm{x}$-axis, log scale). Transcription factors (TF) selected based on Gene Ontology terms (GO:0003700; GO:0006355) (expressed, dashed red line N = 847; not expressed, dashed blue line $N=534$ ) showed higher fractions in both categories when compared to all genes. C: A gene neighborhood was defined as its upstream and downstream regions halfway to the next annotated gene or maximally $50 \mathrm{~Kb}$. The fraction of genes (expressed, red; not-expressed, blue), which contain unexplained intergenic signals within neighborhood is shown. Expressed genes have more of their neighborhoods covered by intergenic signals (top red and blue line). D: Transcription of intergenic sequences increases as nearby gene expression increases. For a selection of genes ( $N=525)$ upregulated from below threshold at E11.5, to above threshold at E13.5 or E15.5, the fraction with nearby intergenic signals is shown as in B at E11.5 (green) and E15.5 (red). A higher fraction of genes with nearby intergenic transcription is seen at E15.5, as the expression increases. 
Table 3 List of differentially expressed long non-coding RNAs, as annotated in UCSC

\begin{tabular}{|c|c|c|c|c|c|c|c|}
\hline \multirow[t]{2}{*}{ Gene Symbol } & \multirow[t]{2}{*}{ UCSC ID } & \multirow[t]{2}{*}{ Location } & \multirow[t]{2}{*}{$\begin{array}{l}\text { PhasCons Score } \\
\text { (mean) }\end{array}$} & \multirow[t]{2}{*}{$\begin{array}{l}\text { Orientation to the closest } \\
\text { coding gene }\end{array}$} & \multirow[t]{2}{*}{$\begin{array}{l}\text { Closest Coding } \\
\text { Gene }\end{array}$} & \multicolumn{2}{|c|}{$\begin{array}{l}\text { Differential Gene } \\
\text { Expression }\end{array}$} \\
\hline & & & & & & $\begin{array}{l}\text { E13.5 vs } \\
\text { E11.5 }\end{array}$ & $\begin{array}{l}\text { E15.5 vs } \\
\text { E13.5 }\end{array}$ \\
\hline AK019125 & uc007byu.1 & Chr1 & 0.08663 & head-to-head & Arl4c & 1 & -1 \\
\hline $\begin{array}{l}\text { AK003315 } \\
\text { (Myeov2) }\end{array}$ & uc007cbq.1 & Chr1 & 0.05824 & head-to-tail & Otos & -1 & 0 \\
\hline AK014513 & uc007eem.1 & Chr1 & 0.10996 & tail-to-head & Camk1g & 1 & 1 \\
\hline AK082757 & uc008iue.1 & Chr2 & 0.06682 & tail-to-head & Btbd14a & 0 & 1 \\
\hline AK007459 & uc008iuf.1 & Chr2 & 0.27284 & head-to-head & Btbd14a & 0 & 1 \\
\hline C130021l20Rik & uc008jhz.1 & Chr2 & 0.13674 & head-to-head & $\operatorname{Lm} \times 1 b$ & 0 & -1 \\
\hline AK030275 & uc008|tx.1 & Chr2 & 0.13798 & head-to-tail & Sid470 & 0 & -1 \\
\hline BC048556 & uc008mgc.1 & Chr2 & 0.21847 & tail-to-head & Nphp1 & 1 & 1 \\
\hline AK007971 & uc008nqb.1 & Chr2 & 0.09166 & tail-to-tail & Lbp & 0 & -1 \\
\hline AK012506 & uc008onz.1 & Chr3 & 0.34076 & antisense & Zfh $\times 4$ & 0 & -1 \\
\hline AK007373 & uc008rbp.1 & Chr3 & 0.09312 & head-to-head & Dph5 & -1 & 0 \\
\hline $\begin{array}{l}\text { BC096391 } \\
\text { (Snhg8) }\end{array}$ & uc008rfm.1 & Chr3 & 0.20019 & tail-to-tail & Prss12 & 0 & -1 \\
\hline AK080292 & uc008rzc.1 & Chr4 & 0.17915 & head-to-head & Ccne2 & 0 & -1 \\
\hline $\begin{array}{l}\text { NR_003270 } \\
\text { (Snhg3) }\end{array}$ & uc008vbf.1 & Chr4 & 0.33261 & head-to-tail & Phactr4 & -1 & -1 \\
\hline AK083203 & uc008wbz.1 & Chr4 & 0.09120 & haid-to-head & Prdm16 & 0 & -1 \\
\hline AK012278 & uc008xth.1 & Chr5 & 0.22535 & head-to-head & Usp46 & -1 & 0 \\
\hline 2610001J05Rik & uc009ayt.1 & Chr6 & 0.13108 & tail-to-head & Gpr85 & 1 & 0 \\
\hline AK002748 & uc009bxj.1 & Chr6 & 0.05646 & head-to-head & $\mathrm{Nfe} 2 \mathrm{l3}$ & 1 & 0 \\
\hline AK033508 & uc009byt.1 & Chr6 & 0.19038 & head-to-head & Hoxa13 & -1 & -1 \\
\hline AK039589 & uc009cib.1 & Chr6 & 0.08391 & head-to-tail & St3gal5 & 1 & -1 \\
\hline AK170805 & uc009ejz.1 & Chr6 & 0.11942 & head-to-head & Tas2r140 & 1 & 0 \\
\hline AK011885 & uc009ely.1 & Chr6 & 0.28077 & head-to-head & Atf7ip & 1 & 0 \\
\hline $\mathrm{H} 19$ & uc009kob.1 & Chr7 & 0.25529 & tail-to-tail & Mrpl23 & -1 & 1 \\
\hline AK134636 & uc009las.1 & Chr8 & 0.13898 & tail-to-head & 4930467E23Rik & 1 & 0 \\
\hline Phxr4 & uc009odu.1 & Chr9 & 0.09209 & tail-to-head & Maml2 & 1 & 0 \\
\hline BC003348 & uc009oek.1 & Chr9 & 0.66034 & head-to-tail & Sesn3 & 0 & -1 \\
\hline AK165129 & uc007htj.1 & Chr11 & 0.37820 & head-to-head & Morc2a & 1 & -1 \\
\hline AK162965 & uc007jdh.1 & Chr11 & 0.18394 & head-to-head & Mrp155 & 1 & -1 \\
\hline AK079857 & uc007kol.1 & Chr11 & 0.06137 & head-to-tail & Slfn3 & 1 & -1 \\
\hline BC037520 & uc007mmx.1 & Chr11 & 0.10152 & tail-to-head & $\operatorname{Sec} 1411$ & 1 & 1 \\
\hline AK164256 & uc007msb.1 & Chr11 & 0.19866 & head-to-head & Bahcc1 & 0 & -1 \\
\hline 1700012B15Rik & uc007mwg.1 & Chr12 & 0.64135 & tail-to-tail & Rab10 & 0 & 1 \\
\hline AK035058 & uc007nvz.1 & Chr12 & 0.30950 & tail-to-tail & Six 1 & 0 & -1 \\
\hline AF498300 (Rian) & uc007paz.1 & Chr12 & 0.05280 & head-to-head & Rt11 & 1 & 1 \\
\hline BC025054 & uc007pyi.1 & Chr13 & 0.11581 & head-to-tail & Sox4 & 1 & -1 \\
\hline AK087718 & uc007pip.1 & Chr13 & 0.15192 & tail-to-head & Gdi2 & -1 & -1 \\
\hline AK021143 & uc007qip.1 & Chr13 & 0.06779 & head-to-head & Fam120a & 1 & -1 \\
\hline AK020502 & uc007qlx.1 & Chr13 & 0.09589 & head-to-head & Spin1 & 0 & -1 \\
\hline AK029385 & uc007vaf.1 & Chr14 & 0.20634 & tail-to-head & Slc15a1 & -1 & 0 \\
\hline AK011684 & uc007vbc.1 & Chr14 & 0.25107 & head-to-head & Zic2 & -1 & 0 \\
\hline DQ715667 & uc007wbs.1 & Chr15 & 0.44104 & tail-to-tail & Chrac1 & -1 & 1 \\
\hline AK085438 & uc007wpq.1 & Chr15 & 0.10360 & tail-to-head & Rac2 & 1 & -1 \\
\hline AK005956 & uc007zwv.1 & Chr16 & 0.13992 & head-to-head & 1110004E09Rik & -1 & 1 \\
\hline AK004150 & uc008cek.1 & Chr17 & 0.09964 & head-to-tail & Hspa1b & 0 & -1 \\
\hline
\end{tabular}


Table 3 List of differentially expressed long non-coding RNAs, as annotated in UCSC (Continued)

\begin{tabular}{|c|c|c|c|c|c|c|c|}
\hline Malat1 & uc008gfj.1 & Chr19 & 0.34059 & tail-to-head & Scyl1 & 1 & 0 \\
\hline Neat1 & uc008gfk.1 & Chr19 & 0.18959 & head-to-tail & Frmd8 & 1 & 1 \\
\hline AK051045 & uc008gmk.1 & Chr19 & 0.23573 & head-to-head & Slc3a2 & 0 & -1 \\
\hline AK030946 & uc009tdn.1 & ChrX & 0.04767 & head-to-tail & 2610018G03Rik & 1 & -1 \\
\hline AK008724 & uc009ura.1 & ChrX & 0.21428 & head-to-head & Kctd12b & 1 & 0 \\
\hline
\end{tabular}

' 1 ' means that the lincRNA is upregulated between the two stages considered, whereas ' -1 ' indicated a down-regulation. The orientations with respect to the closest coding genes, as indicated, were not related to the distance between them. Conservation scores (PhasCons) indicate mean values, as determined from the alignments of 29 vertebrate genomes with the mouse (downloaded from UCSC).

The expression of four long ncRNAs was examined in some details by in situ hybridization (Figure 4), both to validate the transcriptome data and because these ncRNAs are localised at the vicinity of genes known to be relevant for limb development. For instance, the important limb developmental regulator $L m \times 1 b$ gene is surrounded by several intergenic transcripts, in particular by a well-described bi-directional transcript along its promoter region ([26]; Figure 4A). The hybridization pattern of this ncRNA showed a nearly identical spatial distribution, in dorsal limb mesenchyme, and its expression dynamics closely matched that of $L m x 1 b .60 \mathrm{~kb}$ upstream of $L m \times 1 b$, we found a differentially expressed transcript, which may as well be an alternative start site for this gene since it is transcribed from the same DNA strand and expressed in an identical dorsal mesenchyme domain, even though it was more dramatically downregulated at E15.5 than $L m \times 1 b$, as validated by qPCR. This example illustrates the difficulty to discriminate between genuine ncRNAs and parts of known transcription units located nearby.

Two bidirectional non-coding transcripts were also examined in some details by in situ hybridization, one starting very close from the start site of the Hand2 gene, whereas the other one starts at approximately 5 kb from the Prdm16 TSS (Figure 4B, D). Here again, expression patterns were nearly identical between the ncRNAs and their 'host' genes. Both Hand2 and the associated ncRNA were expressed in a patch on the posterior side of the developing limb bud, whereas the $\operatorname{Prdm} 16$ pair was transcribed strongly in distal autopod cells, in particular in growing digits. In both cases, it is thus likely that the transcription of these ncRNAs was achieved by regulatory modules used to control the genes located nearby (or vice-versa).

The fourth case selected as an example is at the Sox4 locus. Sox4 is a rather small gene, located on chromosome 13 and surrounded by large gene deserts, which display series of significant transcript signals during limb development. Several such peaks were observed both in $5^{\prime}$ of the gene, where they matched small bidirectional transcripts (not shown), and in 3' where up to more than $400 \mathrm{~kb}$ large unspliced ncRNAs were scored (annotated). We focused our attention on a peak of transcriptional activity located approximately $50 \mathrm{~kb}$ downstream from the Sox4 3' UTR and tested it by in situ hybridization (Figure 4C). Again, the expression pattern was globally that of Sox4, though with some differences, in particular in the developing major body axis. Expression patterns in the limbs were similar to one another, suggesting once more that this ncRNA was under the control of the regulations acting over Sox4.

ncRNAs appear to be involved in diverse biological processes, as exemplified by the association of some lncRNAs with gene silencing (e.g. $[8,27])$. Our results indicate that these RNA species are frequently coupled either to actively transcribed regions, or to enhancer regions controlling genes of importance for developmental processes $[28,29]$. Accordingly, it is often unclear as to whether these transcripts result from a by-stander effect, i.e. by recruiting the activity of potent enhancer sequences located nearby, or if their control sequences evolved partly or entirely due to their own functional outcomes.

\section{Clustered genes and intergenic transcription}

Gene clusters usually comprise structurally and/or functionally related genes that may have arisen via ancestral gene duplication events. Clustered genes often share some of their regulatory controls, either due to the existence of global regulatory modules controlling several genes at once, or because duplications also included some target sequences for upstream factors. On the other hand, gene duplication events certainly favored some functional divergence. For example, a duplication event generated the $G d f 10 / G d f 2$ pair of transcription factors on chromosome 14; while Gdf10 is differentially expressed in the developing limb (up-regulated from E11.5 toward E13.5), Gdf2 is not expressed there. In contrast, both the Zic2 and Zic5 genes, also produced by a late duplication, are differentially expressed with the same dynamics (down-regulated from E11.5 toward E13.5), whereas the paralogous genes Zic1 and Zic4 were scored as non-expressed. Another case is the Iroquois gene family, with both the $\operatorname{Ir} x 3 / \operatorname{Ir} x 5$ and $\operatorname{Ir} x 1 / \operatorname{Ir} x 2$ pairs scored as being expressed, whereas the distal genes Irx 4 and Irx6 were not detected. Interestingly, in the cases of Zic5, Irx2 and Irx5, promoter-associated bi- 


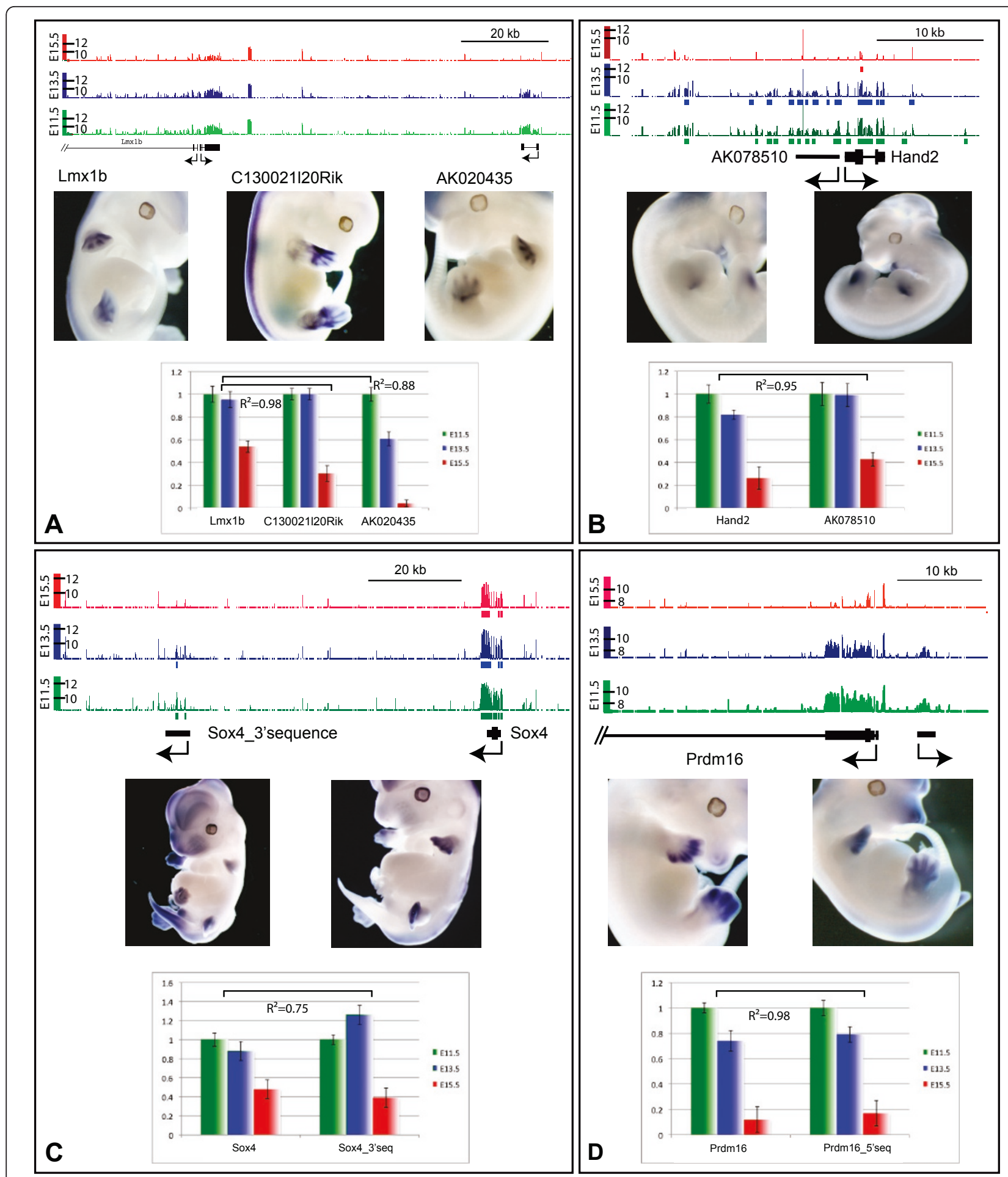

Figure 4 In situ hybridizations of selected intergenic transcripts. The charts show relative amounts of transcripts as measured by qPCR at E11.5 (green), E13.5 (blue) or E15.5 (red). Expression levels at day E11.5 are normalized to 1. Correlation coefficients $\left(\mathrm{R}^{2}\right)$ indicate the relative correlations between the expression of both the protein coding genes and the associated intergenic transcripts. Tiling array data (scales in log 2 on $Y$-axes) and genomic maps are also shown. A. $L m \times 1 b$ gene and associated upstream region, with bidirectional transcripts annotated here as Lmx1b and C130021/20Rik. A potential alternative start site for $L m \times 16,60 \mathrm{~kb}$ upstream (AK020435) shows the same expression specificity. B. Bidirectional transcription at the Hand2 locus, with both Hand2 and the AK078510 transcript displaying related patterns in the posterior margin of developing forelimbs. C. An as yet unknown intergenic transcript (BC025054) located $50 \mathrm{~kb}$ downstream of the Sox4 locus, on the same strand of chromosome 13, which is expressed like Sox4, suggesting it may be a remote $3^{\prime}$ exon of this gene. D: Bidirectional transcription at the Prdm 16 locus on chromosome 4, with an as yet unknown transcript mimicking Prdm16 expression in the E12.5 digital plate. 
directional transcripts were also detected (AK011684, $A K 086211$ and $A K 017076$, respectively). Other examples for clustered expression of transcription factors are the $M y f 5 / 6$ muscle-specific genes [30], which were up-regulated during differentiation, or the $C b x 2 / 8 / 4$ triplet, where $C b x 2$ was down-regulated in older limb buds, whereas $C b x 8$ and $C b x 4$ were expressed steadily throughout the three stages.

The four Hox gene clusters have been used as models for the coordinated regulation of expression in a variety of derivatives, including in developing limbs. In particular, the HoxA and HoxD clusters, which contain 11 and 9 genes, respectively, have essential functions during both hind- and forelimb development [31]. In both gene clusters, we observed transcription profiles in agreement with published datasets, showing a global decrease in intensity in E15.5 limb buds, mostly reflecting the cell type specialization of gene expression and hence a severe dilution effect when compared to the general expression in mesenchymal cells observed in E11.5. Extensive intergenic transcription was scored, whose expression profiles followed those of the surrounding Hox genes (Additional file 7). In the case of $H o x D$, three such intergenic regions encoded by the same strand as all Hoxd genes were tested by in situ hybridization. They all showed expression patterns virtually identical to those of the closest Hoxd genes, suggesting that they may be expanded 3' UTRs (additional file 7). In the case of Hoxa13, a bi-directional transcript was also scored, yet its expression profile was not related to that of Hoxa13. Recently, this transcript (AK033508) was named HOTTIP and shown to be involved in the regulation of HoxA cluster genes in cultured cells [32].

Finally, the largest gene clusters differentially expressed in developing limbs were associated with the skin developmental programs, including the keratin gene complexes on chromosome 11 and 15, or the Sprrb and S100a genes on chromosome 3, with a dominant expression at E15.5. These latter genes are part of the 'epidermal differentiation complex', spanning nearly $3 \mathrm{Mb}$ and composed of members of different gene families clustered together [33-35]; (data not shown).

\section{Imprinted gene clusters and associated ncRNAs}

More than 100 imprinted genes have been reported in the human genome, organized into distinct and large chromosomal regions http://www.har.mrc.ac.uk/ research/genomic_imprinting/[36]. Imprinted gene clusters usually involve genes unrelated in structure or function, and in many cases they contain one or more ncRNAs that may regulate gene expression in cis (long ncRNAs) or in trans (miRNAs, C/D small nucleolar RNAs). A well-known example of a complex imprinted gene cluster is the Dlk1-Gtl2 locus on chromosome 12 .
The whole gene array located on the positive DNA strand seems to be up-regulated in limbs between day 11.5 and 13.5, with the most significant change observed for Gtl2 (Meg3) and the Rian non-coding RNAs (Additional file 7). Dio3, lying at the very 3' end of the complex was silent (not shown). The miRNA cluster, embedded within and in 5' outside the non-coding RNA Mirg, also gave a positive signal, suggesting they may be part of a larger transcript. Amongst the gene members of the cluster, Gtl2 was shown to be important during embryonic development [37], as deletion of the maternal allele resulted in perinatal lethality and skeletal muscle defects [38].

Other imprinted genes relevant for limb and/or bone development were found expressed, including the GnasNespas locus, important for cartilage development [39] or $C d k n 1 c$, within the Kcnq1 imprinted cluster [40], as well as the Igf2-H19 cluster, necessary for skeletal development and ossification [41,42]. Plagl1 (Zac1), a gene encoding a zinc-finger transcription factor, which regulates other imprinted genes and is also required for bone ossification [43], was progressively up-regulated, indicating the potential importance of imprinted genes during limb development.

\section{Conclusions}

These datasets provide a valuable resource, either for research projects involving gene expression and regulation in developing mouse limbs, or as examples of tissue-specific, genome-wide transcriptional activities. More specifically, we found that expression signals are frequently confined to known transcript boundaries but tend to extend past the 3'ends of genes. Also, about 40 percent of known genes were found expressed in limbs of $11.5,13.5$ and 15.5 days old foetuses. We observed extensive intronic transcription, since about 50 percent of expressed genes also contained signals covering intronic sequences. After filtering, more than 4,000 intergenic regions remained clearly transcribed during the three developmental stages. Finally, we identified 49 long ncRNAs that were differentially expressed in our samples.

\section{Methods}

\section{Sampling and microarray hybridization}

C57Bl/6J embryonic forelimbs at stages 11.5 (20 limbs), 13.5 (8 limbs) and 15.5 (2 limbs) were dissected along the body wall and kept in RNAlater. Total RNA was extracted using RNeasy-mini columns (Qiagen), DNaseI digested on-column, treated with RNase free DNase I once more (Roche), and further processed according to the Affymetrix GeneChip WT Double-Stranded Target Assay manual. Briefly, ribosomal RNAs were depleted and samples were reverse transcribed. These cDNAs 
served as templates for cRNA synthesis (amplification), which were then reverse transcribed again. The products were fragmented, labeled and hybridized onto Genechip Mouse Tiling $1.1 \mathrm{R}$ arrays. The qualities of all initial steps were monitored by Bioanalyzer 2100 (Agilent). All microarray-related experiments were done in duplicate.

All experiences involving animals were carried according to the Swiss law, under the authorization number $1008 / 3482 / 0$ (to DD).

\section{Microarray data processing}

The tiling array set consisted of 14 chips. For each of these chips and each of the six samples (3 time-points $x$ 2 duplicates), standard background correction and quantile normalization was performed; mismatch probes were not used. The correlation of probe intensities $(\log 2)$ on all replicated arrays ranged form 0.84 and 0.91 . Probe coordinates were converted to the July 2007 build of the mouse genome using the UCSC liftOver software http:// hgdownload.cse.ucsc.edu/admin/exe/. For each chromosome on a chip, a summary procedure was then performed as follows. Known gene annotations were downloaded from UCSC http://genome.ucsc.edu/cgibin/hgTables and complemented by miRNA, snRNA and snoRNA annotations from ensemble http://www. ensembl.org. All probes in an exon were considered as a single block. In introns and intergenic regions, blocks were defined using a sliding window of 500 bp width. For each block, a linear model identical to that in the RMA method [44] was used to estimate the three condition-specific expression levels from the six samples. This regression was done after log transformation of the normalized intensity data. Thresholds for expression of exons were defined by the 99th percentile of the distribution of expression levels in the regions annotated as intergenic. Transcripts with $80 \%$ or more expressed exons were considered to be expressed. Genes with 50\% or more expressed exons were considered to be expressed. Small RNAs were only considered if they were larger than $100 \mathrm{bp}$.

\section{Identification of differentially expressed genes}

Differences in expression were calculated by taking the median (log-scale) expression level across the exons of a gene, and then calculating the differences between pairs of experimental conditions. A permutation method was used to define significance thresholds on differential expression: probes were permutated locally along the genome as follows: a random variable drawn from a Gaussian distribution (standard deviation $=1 \mathrm{Mb}$ ) for each probe and was added to the probe coordinates. The probes were then ranked according to their new coordinates. In the permutated dataset, the intensity of a probe was then replaced by the intensity of the probe with equal rank in the randomized coordinates. Five permutated datasets were generated and differential expression levels of genes were calculated as above. Expression differences greater than the 99th percentile or smaller than the first percentile of the expression differences in the combined permutated datasets were considered as significant.

\section{Intronic and intergenic signal analyses}

Intronic signals were defined as continuous regions above threshold of at least $300 \mathrm{bp}$ length and containing no unspotted regions (regions without hybridization probes) of more than $100 \mathrm{bp}$. Intergenic signals were defined as continuous regions above threshold of at least 300 bp lengths. Once identified, intergenic signals were classified as flanking UCSC annotations if they were within $100 \mathrm{bp}$ of a UCSC known gene annotation. Wherever possible, intergenic signals not classified as flanking UCSC annotations were identified from Ensembl annotation, differentiating between Ensembl overlap, where an overlap of at least 200 bp existed between an intergenic signal and an Ensembl annotation, and Ensembl flanking, where the intergenic signal was within $100 \mathrm{bp}$ of the Ensembl annotation. The remaining signals were considered and termed unexplained. expressed intergenic regions (orange) at E11.5 were annotated as 'close to' an expressed gene (red), 'close to' a non-expressed (blue), 'not close to' a gene (green). For Figure 3, the distance cut-off to assign 'close' varied form $1 \mathrm{~Kb}$ to $1 \mathrm{Mb}$ ( $\mathrm{x}$-axis) using bin size increasing by $1 \mathrm{~Kb}$ from $1 \mathrm{~Kb}$ to $20 \mathrm{~Kb}$, by $10 \mathrm{~Kb}$ from $20 \mathrm{~Kb}$ to $100 \mathrm{~Kb}$ and by $100 \mathrm{~Kb}$ from $100 \mathrm{~Kb}$ to $1 \mathrm{Mb}$.

\section{Conservation of ncRNA sequences}

PhastCons conservation scores [45] were downloaded from UCSC website using 29 vertebrate genomes alignments with the Mouse (mm9-PhasCons30way). Mean conservation scores were calculated for genomic regions covering the selected ncRNAs.

Whole mount in situ hybridization and quantitative PCR Whole mount in situ hybridizations were performed according to standard protocol. Amplified cDNA products were ligated into pTopo-BluntII (Invitrogen) vector. Digoxigenin (Roche) labeled riboprobes were produced using T7 or Sp6 RNA polymerase (Promega). Hybridizations were carried out overnight at $68^{\circ} \mathrm{C}$. Hybridizations were detected using NBT/BCIP solutions (Roche). Real-time qPCRs were done in triplicate with Express Sybr GreenER premix (Invitrogen) using CFX96 Real-Time system (Biorad). Primers are compiled in the supplementary material (Additional file 8). 


\section{Data availability}

Raw tiling array data (CEL files) as well as .wig files suitable for display in the UCSC genome browser are available at the Gene Expression Omnibus (GEO) under accession number: GSE27417 http://www.ncbi.nlm.nih. gov/geo/query/acc.cgi?acc=GSE27417.

\section{Additional material}

Additional file 1: Supplementary Table 1: List of identified

differentially expressed genes. Plus 1 indicate up-regulation, minus 1 down-regulation, zero means no change, respectively. The 2 comparisons are shown in parallel columns (i.e. E13.5 minus E11.5 and E15.5 minus E13.5).

Additional file 2: Supplementary Figure 1: Validation of some selected differentially expressed genes by quantitative PCR and/or whole mount in situ hybridization. A. Bar charts show relative gene expression levels, with the measured transcript level at E11.5 taken as a reference ('1'). A: Relative expression for Hoxd8 to Hoxd13; Hoxc6; Cbln1; Lhx9 and Aff3. B: Relative gene expression of bone specific marker genes that were up-regulated at E15.5: Col1a1; Ibsp; Spp1. C: Relative gene expression of Col2a1; Aldh1a2; Osterix (Sp7); Noggin and Tmem8c. Steadystate levels of selected RNAs were assessed at the three stages (in green for E11.5; blue for E13.5 and red for E15.5). While expression of control Hoxd genes expectedly decreased at E15.5 (probably due to the decrease of mesenchymal cell mass at this stage), expression of genes specific for late stages of cartilage differentiation was markedly increased. D: In situ hybridizations of selected differentially expressed genes. Genes were selected according to their various expression dynamics: Lhx9 and Aff3 were progressively down-regulated during development (i.e. from E11.5 to E15.5); Tmem8c was progressively up-regulated; Noggin tend to upregulated between E11.5 and E13.5 but down-regulated later (see qPCR data above) while Hoxc6 and Cbln 1 were expressed only at E11.5 then rapidly down-regulated. Gene symbols and developmental stages are indicated.

Additional file 3: Supplementary Figure 2: Gene ontology analysis of differentially expressed genes using GOMiner. Hierarchical

clustering of enriched gene ontology terms (biological functions) characterizing differentially expressed genes. The settings were: evidence level of 3, minimum cluster size of 5 and maximum FDR of 0.05. A, B. Clusters of enriched $\mathrm{GO}$ terms for those genes differentially expressed, either between E13.5 and E11.5 (A) or between E15.5 and 13.5 (B). X-axis indicates $\mathrm{GO}$ categories, Y-axis for differentially expressed genes. Red areas indicate up-regulated genes, whereas the green areas represent down-regulated genes those are sharing the same functional categories.

Additional file 4: Supplementary Table 2: Top 50 cluster categories ranked by p-values (calculated from Fisher's exact test) and GO term enrichments (minimum 2 fold). The left panel shows the enriched categories for those genes that are differentially expressed between E11.5 and E13.5, whereas the panel in the right is for those genes differentially expressed between E13.5 and E15.5.

Additional file 5: Supplementary Table 3: List of differentially expressed genes that were previously associated with short or abnormal limb phenotype, based on Mammalian Phenotype Ontology.

Additional file 6: Supplementary Table 4: Gene model categories of intergenic transcripts identified via Ensembl. All intergenic regions were screened for potential overlaps with Ensembl annotation. Identified genes were classified according to Ensembl gene model categories.

Additional file 7: Supplementary Figure 3: Intergenic transcription within gene clusters. A. Tiling array gene expression data along the entire HoxD gene cluster on chromosome 2. Transcription is scored outside annotated transcription units, at least in three regions; igs1, igs2 and igs3 (black bars). B: In situ hybridizations on E12.5 embryos, with three different probes corresponding to these transcribed intergenic regions. igs 1 lies between Hoxd11 and Hoxd12, whereas both igs2 and igs3 are located between Hoxd10 and Hoxd11. The transcription profiles of these RNAs follow the general logic of the gene cluster and thus resemble the expression of the neighboring genes. C. Tiling array expression data along the entire HoxA gene cluster on chromosome 6, showing intergenic transcription, also from the opposite DNA strand (Hoxal1as and AK033508). D. Transcription profiles at the D/k1-Gt/2 imprinted gene cluster on chromosome 12. The vertical black bars (on the 'positive' strand) point either to C/D small RNAs, mapping around Rt $/ 1$, or to miRNAs located around Mirg. The small transcript overlapping with $R$ t/1 is referred to as Rt/1-antisense transcript. All scales on tiling array's $Y$-axes are in $\log _{2}$.

Additional file 8: Supplementary Table 5: List of oligonucleotide sequences used for quantitative PCR. (A) or cloning procedures (B). All sequences are indicated from 5-prime to 3-prime.

\section{Abbreviations}

AER: Apical Ectodermal Ridge; AP: Anterior to Posterior; DV: Dorsal to Ventral; ncRNAs: Non-coding RNAs; IncRNAs: Long non-coding RNAs; TSS:

Transcription start site; UTR: Untranslated region; TF: Transcription factor.

\section{Acknowledgements and Funding}

We thank the members of the NCCR genomics platform for their help with tiling arrays as well as members of the Duboule laboratories for discussions. This work was supported by funds from the Ecole Polytechnique Fédérale, Lausanne, the University of Geneva, the Swiss National Research Fund, the National Research Center (NCCR) "Frontiers in Genetics", the EU program "Crescendo" and the ERC grant Systems Hox.ch (to D.D.).

\section{Author details}

'School of Life Sciences, Ecole Polytechnique Fédérale, Station 19, Lausanne, 1215 Switzerland. 'Swiss Institute of Bioinformatics, Batiment Génopode, University of Lausanne, 1215 Switzerland. ${ }^{3}$ Department of Genetics and Evolution, University of Geneva Quai Ernest Ansermet, 30, Geneva, 1211 Switzerland.

\section{Authors' contributions}

IG helped design and carried out the experiments and drafted the manuscript, BS analyzed the data, FN supervised data analysis and interpretation and DD designed the experiments and wrote the paper. All authors helped writing the article.

\section{Competing interests}

The authors declare that they have no competing interests.

Received: 4 May 2011 Accepted: 29 July 2011 Published: 29 July 2011

\section{References}

1. Niswander L: Pattern formation: old models out on a limb. Nat Rev Genet 2003, 4:133-143.

2. Zeller R, Lopez-Rios J, Zuniga A: Vertebrate limb bud development: moving towards integrative analysis of organogenesis. Nat Rev Genet 2009, 10:845-858.

3. Tabin C, Wolpert L: Rethinking the proximodistal axis of the vertebrate limb in the molecular era. Genes Dev 2007, 21:1433-1442.

4. Gerard M, Chen JY, Gronemeyer H, Chambon P, Duboule D, Zakany J: In vivo targeted mutagenesis of a regulatory element required for positioning the Hoxd-11 and Hoxd-10 expression boundaries. Genes Dev 1996, 10:2326-2334.

5. Cretekos CJ, Wang Y, Green ED, Martin JF, Rasweiler JJt, Behringer RR: Regulatory divergence modifies limb length between mammals. Genes Dev 2008, 22:141-151.

6. Richardson MK, Gobes SM, van Leeuwen AC, Polman JA, Pieau C, SanchezVillagra MR: Heterochrony in limb evolution: developmental mechanisms and natural selection. J Exp Zool B Mol Dev Evol 2009, 312:639-664.

7. Amaral PP, Mattick JS: Noncoding RNA in development. Mamm Genome 2008, 19:454-492.

8. Khalil AM, Guttman M, Huarte M, Garber M, Raj A, Rivea Morales D, Thomas K, Presser A, Bernstein BE, van Oudenaarden A, Regev A, Lander ES, 
Rinn JL: Many human large intergenic noncoding RNAs associate with chromatin-modifying complexes and affect gene expression. Proc Natl Acad Sci USA 2009, 106:11667-11672.

9. Mercer TR, Dinger ME, Mattick JS: Long non-coding RNAs: insights into functions. Nat Rev Genet 2009, 10:155-159.

10. Ponting $\mathrm{CP}$, Oliver $\mathrm{PL}$, Reik W: Evolution and functions of long noncoding RNAs. Cell 2009, 136:629-641

11. Pang KC, Frith MC, Mattick JS: Rapid evolution of noncoding RNAs: lack of conservation does not mean lack of function. Trends Genet 2006, 22:1-5.

12. Feng J, Bi C, Clark BS, Mady R, Shah P, Kohtz JD: The Evf-2 noncoding RNA is transcribed from the Dlx-5/6 ultraconserved region and functions as a Dlx-2 transcriptional coactivator. Genes Dev 2006, 20:1470-1484.

13. Sleutels F, Zwart R, Barlow DP: The non-coding Air RNA is required for silencing autosomal imprinted genes. Nature 2002, 415:810-813.

14. Koerner MV, Pauler FM, Huang R, Barlow DP: The function of non-coding RNAs in genomic imprinting. Development 2009, 136:1771-1783.

15. Martin P: Tissue patterning in the developing mouse limb. Int I Dev Biol 1990, 34:323-336.

16. van Bakel H, Nislow C, Blencowe BJ, Hughes TR: Most "dark matter" transcripts are associated with known genes. PLoS Biol 2010, 8:e1000371.

17. Matsuda K, Miura E, Miyazaki T, Kakegawa W, Emi K, Narumi S, Fukazawa Y, Ito-Ishida A, Kondo T, Shigemoto R, Watanabe M, Yuzaki M: Cbln1 is a ligand for an orphan glutamate receptor delta2, a bidirectional synapse organizer. Science 2010, 328:363-368.

18. Zeeberg BR, Feng W, Wang G, Wang MD, Fojo AT, Sunshine M, Narasimhan S, Kane DW, Reinhold WC, Lababidi S, Bussey KJ, Riss J, Barrett JC, Weinstein JN: GoMiner: a resource for biological interpretationof genomic and proteomic data. Genome Biol 2003, 4:R28.

19. Mikkelsen TS, Ku M, Jaffe DB, Issac B, Lieberman E, Giannoukos G, Alvarez $P$, Brockman W, Kim TK, Koche RP, Lee W, Mendenhall E, O'Donovan A, Presser A, Russ C, Xie X, Meissner A, Wernig M, Jaenisch R, Nusbaum C, Lander ES, Bernstein BE: Genome-wide maps of chromatin state in pluripotent and lineage-committed cells. Nature 2007, 448:553-560.

20. Meissner A, Mikkelsen TS, Gu H, Wernig M, Hanna J, Sivachenko A, Zhang X, Bernstein BE, Nusbaum C, Jaffe DB, Gnirke A, Jaenisch R, Lander ES: Genome-scale DNA methylation maps of pluripotent and differentiated cells. Nature 2008, 454:766-770.

21. Tian B, Hu J, Zhang H, Lutz CS: A large-scale analysis of mRNA polyadenylation of human and mouse genes. Nucleic Acids Res 2005, 33:201-212.

22. Spitz F, Duboule D: Global control regions and regulatory landscapes in vertebrate development and evolution. Adv Genet 2008, 61:175-205.

23. Ku M, Koche RP, Rheinbay E, Mendenhall EM, Endoh M, Mikkelsen TS, Presser A, Nusbaum C, Xie XH, Chi AS, Adli M, Kasif S, Ptaszek LM, Cowan CA, Lander ES, Koseki H, Bernstein BE: Genomewide Analysis of PRC1 and PRC2 Occupancy Identifies Two Classes of Bivalent Domains. Plos Genetics 2008, 4:e1000242.

24. Sunwoo H, Dinger ME, Wilusz JE, Amaral PP, Mattick JS, Spector DL: MEN epsilon/beta nuclear-retained non-coding RNAs are up-regulated upon muscle differentiation and are essential components of paraspeckles. Genome Res 2009, 19:347-359.

25. Carninci P, Kasukawa T, Katayama S, Gough J, Frith MC, Maeda N, Oyama R, Ravasi T, Lenhard B, Wells C, Kodzius R, Shimokawa K, Bajic VB, Brenner SE, Batalov S, Forrest AR, Zavolan M, Davis MJ, Wilming LG, Aidinis V, Allen JE, Ambesi-Impiombato A, Apweiler R, Aturaliya RN, Bailey TL, Bansal M, Baxter L, Beisel KW, Bersano T, Bono $\mathrm{H}$, et al: The transcriptional landscape of the mammalian genome. Science 2005, 309(5740):1559-1563.

26. Holmes G, Crooijmans R, Groenen M, Niswander L: ALC (adjacent to LMX1 in chick) is a novel dorsal limb mesenchyme marker. Gene Expr Patterns 2003, 3:735-741.

27. Rinn JL, Kertesz M, Wang JK, Squazzo SL, Xu X, Brugmann SA Goodnough LH, Helms JA, Farnham PJ, Segal E, Chang HY: Functional demarcation of active and silent chromatin domains in human HOX loci by Noncoding RNAs. Cell 2007, 129:1311-1323.

28. Kim TK, Hemberg M, Gray JM, Costa AM, Bear DM, Wu J, Harmin DA, Laptewicz M, Barbara-Haley K, Kuersten S, Markenscoff-Papadimitriou E, Kuhl D, Bito H, Worley PF, Kreiman G, Greenberg ME: Widespread transcription at neuronal activity-regulated enhancers. Nature 2010, 465:182-187.
29. Rada-Iglesias A, Bajpai R, Swigut T, Brugmann SA, Flynn RA, Wysocka J: A unique chromatin signature uncovers early developmental enhancers in humans. Nature 2011, 470:279-283.

30. Braun T, Buschhausen-Denker G, Bober E, Tannich E, Arnold HH: A novel human muscle factor related to but distinct from MyoD1 induces myogenic conversion in 10T1/2 fibroblasts. EMBO J 1989, 8:701-709.

31. Zakany J, Duboule D: The role of Hox genes during vertebrate limb development. Current Opinion in Genetics \& Development 2007, 17:359-366.

32. Wang KC, Yang YW, Liu B, Sanyal A, Corces-Zimmerman R, Chen $Y$, Lajoie BR, Protacio A, Flynn RA, Gupta RA, Wysocka J, Lei M, Dekker J, Helms JA, Chang HY: A long noncoding RNA maintains active chromatin to coordinate homeotic gene expression. Nature 2011, 472:120-124.

33. Marshall D, Hardman MJ, Nield KM, Byrne C: Differentially expressed late constituents of the epidermal cornified envelope. Proc Natl Acad Sci USA 2001, 98:13031-13036.

34. Marenholz I, Zirra M, Fischer DF, Backendorf C, Ziegler A, Mischke D: Identification of human epidermal differentiation complex (EDC)encoded genes by subtractive hybridization of entire YACs to a gridded keratinocyte cDNA library. Genome Res 2001, 11:341-355.

35. Sproul D, Gilbert N, Bickmore WA: The role of chromatin structure in regulating the expression of clustered genes. Nat Rev Genet 2005, 6:775-781.

36. Williamson CM, Blake A, Thomas S, Beechey CV, Hancock J, Cattanach BM, Peters J: Mouse Imprinting Data and References. MRC Harwell, Oxfordshire; 2011 [http://www.har.mrc.ac.uk/research/genomic_imprinting/].

37. Schuster-Gossler K, Bilinski P, Sado T, Ferguson-Smith A, Gossler A: The mouse Gtl2 gene is differentially expressed during embryonic development, encodes multiple alternatively spliced transcripts, and may act as an RNA. Dev Dyn 1998, 212:214-228.

38. Zhou Y, Cheunsuchon P, Nakayama Y, Lawlor MW, Zhong Y, Rice KA, Zhang L, Zhang X, Gordon FE, Lidov HG, Bronson RT, Klibanski A: Activation of paternally expressed genes and perinatal death caused by deletion of the Gt12 gene. Development 2010, 137:2643-2652

39. Sakamoto A, Chen M, Kobayashi T, Kronenberg HM, Weinstein LS: Chondrocyte-specific knockout of the $G$ protein $G(s)$ alpha leads to epiphyseal and growth plate abnormalities and ectopic chondrocyte formation. J Bone Miner Res 2005, 20:663-671.

40. Yan Y, Frisen J, Lee MH, Massague J, Barbacid M: Ablation of the CDK inhibitor p57Kip2 results in increased apoptosis and delayed differentiation during mouse development. Genes Dev 1997, 11:973-983.

41. Eggenschwiler J, Ludwig T, Fisher P, Leighton PA, Tilghman SM, Efstratiadis A: Mouse mutant embryos overexpressing IGF-II exhibit phenotypic features of the Beckwith-Wiedemann and Simpson-GolabiBehmel syndromes. Genes Dev 1997, 11(23):3128-3142.

42. Liu JP, Baker J, Perkins AS, Robertson EJ, Efstratiadis A: Mice carrying null mutations of the genes encoding insulin-like growth factor I (Igf-1) and type 1 IGF receptor (Igf1r). Cell 1993, 75:59-72.

43. Varrault A, Gueydan C, Delalbre A, Bellmann A, Houssami S, Aknin C, Severac D, Chotard L, Kahli M, Le Digarcher A, Pavlidis P, Journot L: Zac1 regulates an imprinted gene network critically involved in the control of embryonic growth. Dev Cell 2006, 11:711-722.

44. Irizarry RA, Hobbs B, Collin F, Beazer-Barclay YD, Antonellis KJ, Scherf U, Speed TP: Exploration, normalization, and summaries of high density oligonucleotide array probe level data. Biostatistics 2003, 4:249-264.

45. Siepel A, Bejerano G, Pedersen JS, Hinrichs AS, Hou M, Rosenbloom K, Clawson H, Spieth J, Hillier LW, Richards S, Weinstock GM, Wilson RK, Gibbs RA, Kent WJ, Miller W, Haussler D: Evolutionarily conserved elements in vertebrate, insect, worm, and yeast genomes. Genome Res 2005, 15:1034-1050.

\section{doi:10.1186/1471-213X-11-47}

Cite this article as: Gyurján et al:: Analysis of the dynamics of limb transcriptomes during mouse development. BMC Developmental Biology 2011 11:47. 